\title{
Modelling thermal flow in the transition regime using a lattice Boltzmann approach
}

\author{
Y.-H. Zhang ${ }^{*}$ X. J. Gu, R. W. BARber and D. R. Emerson \\ Centre for Microfluidics and Microsystems Modelling, Computational Science and Engineering Department, CCLRC \\ Daresbury Laboratory, Warrington, WA4 4AD, UK
}

\author{
PACS 05.10.-a - Computational techniques in statistical physics and nonlinear dynamics \\ PACS 44.10.+i - Heat conduction \\ PACS 47.45.-n-Dynamics of rarefied gases
}

\begin{abstract}
Lattice Boltzmann models are already able to capture important rarefied flow phenomena, such as velocity-slip and temperature jump, provided the effects of the Knudsen layer are minimal. However, both conventional hydrodynamics, as exemplified by the Navier-StokesFourier equations, and the lattice Boltzmann method fail to predict the nonlinear velocity and temperature variations in the Knudsen layer that have been observed in kinetic theory. In the present paper, we propose an extension to the lattice Boltzmann method that will enable the simulation of thermal flows in the transition regime where Knudsen layer effects are significant. A correction function is introduced that accounts for the reduction in the mean free path near a wall. This new approach is compared with direct simulation Monte Carlo data for Fourier flow and good qualitative agreement is obtained for Knudsen numbers up to 1.58.
\end{abstract}

Recent technological progress has enabled major advances in the development of miniaturised devices and this has led to significant interest in trying to understand low-speed rarefied gas flow in the slip and transition regime. Traditional numerical approaches for modelling high-speed rarefied flow, such as the direct simulation Monte Carlo (DSMC) method, are very inefficient for low-speed flows whilst solving Boltzmann's equation directly still remains a formidable challenge. Although conventional hydrodynamic approaches based on the Navier-Stokes-Fourier (NSF) equations are computationally efficient, they quickly break down when the flow enters the transition regime. In contrast, the lattice Boltzmann method has been developed from kinetic theory and has a computational efficiency comparable to NSF methods [1-4]. Moreover, numerous authors have shown that the lattice Boltzmann equation (LBE) can simulate lowspeed micro- and nano-scale flows e.g. [5-21]. To date, most LBE work has focused on capturing velocity-slip under isothermal conditions. However, for the case of planar Couette flow, we have recently demonstrated [22] that a thermal LBE model can produce reasonably accurate heat transfer predictions in the slip-flow regime.

In the transition regime, nonequilibrium effects within the Knudsen layer become increasingly important. To capture flows in the transition regime, current LBE models clearly need to be improved. One approach is through the construction of new LBE schemes that ensure the conser-

*Author to whom correspondence should be addressed. Fax: (+44)1925 603634; Email: Y.Zhang@dl.ac.uk

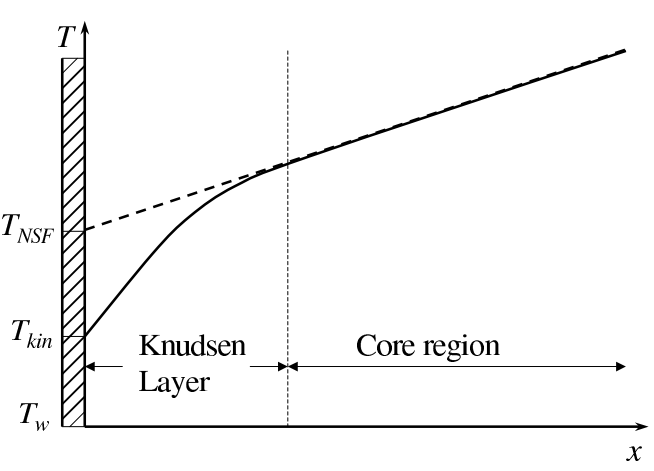

Fig. 1: Schematic diagram of Fourier flow showing the temperature profile in the Knudsen layer for a constant heat flux normal to the wall, where $T_{w}$ represents the wall temperature and $T_{k i n}$ and $T_{N S F}$ correspond to the gas temperatures predicted by kinetic theory and the NSF equations, respectively.

vation of the higher-order velocity moments [23]. However, in the present paper, we propose an alternative treatment that extends the LBE method into the transition regime through the implementation of a Knudsen layer correction. The phenomenological nature of this approach retains the computational efficiency and simplicity of existing LBE codes.

The behaviour of a gas near a solid surface will clearly be affected by the interactions between the molecules and 
the wall. This leads to the formation of a kinetic boundary layer, or Knudsen layer, with a thickness $\sim \mathcal{O}(l)$, where $l$ is the mean free path. In the Knudsen layer, the mean time between collisions is less than in the core region and this will lead to a reduction in the mean free path close to the wall. Conventional hydrodynamic approaches based on the Navier-Stokes-Fourier equations are not appropriate in the Knudsen layer but remain valid in the core region. The gas temperature in the Knudsen layer is markedly different to the predictions obtained from the NSF equations, as shown schematically in fig. 1 .

The effect of the wall on the mean free path has recently been discussed by Guo et al. [24] where the authors proposed a correction function that depended on the Knudsen number. As the authors observed, this approach did not allow the LBE method to capture the effects of the Knudsen layer although there was a slight improvement in the prediction of the slip velocity. However, the presence of a Knudsen layer implies a spatial variation in the mean free path that depends on the distance from the wall. In the present approach we propose that the effective mean free path, $l_{e}$, can be expressed as

$$
l_{e}=\frac{l}{1+\psi(x / l)},
$$

where $x$ is the distance normal to the wall and the mean free path is given by $l=\mu / p \sqrt{\pi R T / 2}$, where $\mu$ is the viscosity, $p$ is the pressure, $T$ is the temperature and $R$ is the gas constant. The correction factor, $\psi(x / l)$, will depend on the distance from the wall and is chosen to be negligible outside the Knudsen layer. The influence of the density and temperature on the effective mean free path is imposed through $l$. Once the correction factor for the Knudsen layer is known, the effective viscosity, $\mu_{e}$, and thermal diffusivity, $\alpha_{e}$, can be determined from

$$
\mu_{e}(x / l)=\frac{\mu}{1+\psi(x / l)},
$$

and

$$
\alpha_{e}(x / l)=\frac{\nu / P r}{1+\psi(x / l)},
$$

where $\nu$ is the kinematic viscosity and $\operatorname{Pr}$ is the Prandtl number. The effective viscosity (2) and thermal diffusivity (3) can readily be used to extend the capability of NSF approaches.

From kinetic theory, the mean free path can be related to the viscosity and the mean molecular velocity, $\bar{c}$, by

$$
\mu=a \rho \bar{c} l
$$

where $\bar{c}=\sqrt{8 R T / \pi}, \rho$ is the density, and $a$ is a constant with an approximate value of 0.499 [25]. For flows involving thermal variations, the viscosity will depend on the temperature, $T$, according to

$$
\mu \propto T^{\omega}
$$

where the value of $\omega$ depends on the molecular interaction model and varies between 0.5 for hard-sphere molecular interactions and 1 for Maxwellian interactions [25]. The influence of any temperature and density variations on the mean free path is therefore given by

$$
\frac{l}{l_{\text {ref }}}=\frac{\rho_{\text {ref }}}{\rho}\left(\frac{T}{T_{\text {ref }}}\right)^{\omega-0.5},
$$

where $l_{\text {ref }}$ and $\rho_{\text {ref }}$ are the mean free path and density at some reference temperature, $T_{r e f}$. The effective mean free path, $l_{e}$, can therefore be written as

$$
\frac{l_{e}}{l_{\text {ref }}}=\frac{1}{1+\psi(x / l)} \frac{\rho_{\text {ref }}}{\rho}\left(\frac{T}{T_{\text {ref }}}\right)^{\omega-0.5} .
$$

The current lack of data makes it difficult to directly evaluate the correction factor, $\psi(x / l)$, for the mean free path. However, we can take advantage of previous work by Lockerby et al. [26] on phenomenological models of the Knudsen layer. For Kramers' problem, the correction factor for the mean free path can be approximated by

$$
\psi(x / l)=\frac{7}{10} e^{-C x / l},
$$

where $C$ is a constant that depends on the governing equations (e.g. NSF, regularised Burnett, BGK-Burnett) [26]. This constant effectively controls the extent of the Knudsen layer and in the present paper we have assumed $C=1$. Equation (8) was developed for isothermal flows where the Knudsen layers do not overlap. As discussed by Chen et $a l$. [27], there is a similarity between turbulence and finite Knudsen number phenomena. Therefore, this Kundsen layer correction has some analogy with the van Driest wall damping function used in turbulence modelling.

In previous work [22], we have shown that the LBE method can predict low-speed thermal rarefied gas flows in the slip-flow regime. The present model, which is based on the work of He et al. [28] and Shi et al. [29], can be summarised as follows:

$$
\begin{array}{r}
f_{k}\left(\mathbf{x}+\mathbf{e}_{k} \delta t, t+\delta t\right)-f_{k}(\mathbf{x}, t)=-\frac{1}{\tau}\left[f_{k}(\mathbf{x}, t)-\right. \\
\left.f_{k}^{e q}(\mathbf{x}, t)\right]+\delta t \frac{\left(e_{k i}-u_{i}\right) F_{i}}{c_{s}^{2} \rho} f_{k}^{e q}(\mathbf{x}, t),
\end{array}
$$

and

$g_{k}\left(\mathbf{x}+\mathbf{e}_{k} \delta t, t+\delta t\right)-g_{k}(\mathbf{x}, t)=-\frac{1}{\tau_{t}}\left[g_{k}(\mathbf{x}, t)-g_{k}^{e q}(\mathbf{x}, t)\right]$,

where $f_{k}(\mathbf{x}, t)$ and $g_{k}(\mathbf{x}, t)$ are the distribution functions at position $\mathbf{x}$ and time $t$ for the number and energy densities, respectively, $f_{k}^{e q}$ and $g_{k}^{e q}$ are the distribution functions at equilibrium, $e_{k i}$ is the lattice velocity, $u_{i}$ is the macroscopic velocity, $c_{s}$ is the lattice speed of sound, $F_{i}$ is the external body force, and $\tau$ and $\tau_{t}$ are the nondimensional relaxation times for the number and energy density distribution functions, respectively. The relationship between 
the two distribution functions is given by

$$
g_{k}=\frac{\left(e_{i}-u_{i}\right)^{2}}{2} f_{k}
$$

Following Shi et al. [29], the viscosity, $\nu$, is given by $(\tau-$ $0.5) c_{s}^{2} \delta t$ and the thermal diffusivity, $\alpha$, is $\left(\tau_{t}-0.5\right) c_{s}^{2} \delta t$. The Prandtl number, $P r$, can then be determined from $(\tau-0.5) /\left(\tau_{t}-0.5\right)$. Once the distribution functions are known, the macroscopic properties can be obtained from

$$
\rho=\int f d e_{i}, \quad \rho u_{i}=\int f e_{i} d e_{i}, \quad \rho \epsilon=\int g d e_{i},
$$

where $\epsilon=D R T / 2$ and $D$ is the number of physical dimensions. If we consider a two dimensional, nine-velocity lattice model (D2Q9), the lattice velocities can be written as

$$
\begin{aligned}
e_{0} & =0 \\
e_{k} & =c(\cos [(k-1) \pi / 2], \sin [(k-1) \pi / 2]), k=1,2,3,4 \\
e_{k} & =\sqrt{2} c(\cos [(k-5) \pi / 2+\pi / 4], \sin [(k-5) \pi / 2 \\
& +\pi / 4]), \quad k=5,6,7,8
\end{aligned}
$$

where $c=\sqrt{3 R T}$ is the rms molecular speed. The equilibrium distribution function for the number density is given by

$$
\begin{array}{r}
f_{k}^{e q}=\rho \omega_{k}\left[1+\frac{e_{k i} u_{i}}{c_{s}^{2}}+\frac{\left(e_{k i} u_{i}\right)^{2}}{2 c_{s}^{4}}-\frac{u_{i} u_{i}}{2 c_{s}^{2}}\right], \quad \omega_{0}=\frac{4}{9} \\
\omega_{k}=\frac{1}{9}, \quad k=1,2,3,4 ; \quad \omega_{k}=\frac{1}{36}, \quad k=5,6,7,8,
\end{array}
$$

and the equilibrium distribution function for the energy density is given by

$$
g_{k}^{e q}=\epsilon f_{k}^{e q} .
$$

To simulate rarefied conditions, the relaxation time of the LBE model is a function of the Knudsen number, $K n$, which relates the mean free path to the characteristic length scale of the flow domain, $H$. For a D2Q9 lattice BGK model, the Knudsen number can be written as $K n=\sqrt{8 / 3 \pi}(\tau-0.5) / N_{H}$, where $N_{H}$ is the number of lattice sites across $H$ [30]. The relaxation time due to the reduction in the mean free path in the Knudsen layer can therefore be written as

$$
\tau=\sqrt{\frac{3 \pi}{8}} \frac{K n N_{H}}{1+\psi(x / l)} \frac{\rho_{\text {ref }}}{\rho}\left(\frac{T}{T_{\text {ref }}}\right)^{\omega-0.5}+0.5,
$$

where $K n=l_{\text {ref }} / H$, and the thermal relaxation time is determined from

$$
\tau_{t}=(\tau-0.5) / \operatorname{Pr}+0.5 \text {. }
$$

It should be noted that the local Knudsen number depends on the temperature and can therefore be evaluated as part of the numerical simulation. One advantage of using a two-distribution function thermal LBE model is that the Prandtl number is no longer fixed.

The kinetic boundary condition proposed by Niu et al. [7] has been used to model the slip velocity at the wall. Assuming the molecular reflections at the wall are fullydiffuse leads to

$$
\begin{array}{r}
\left|\left(\mathbf{e}_{k}-\mathbf{u}_{w}\right) \cdot \mathbf{n}\right| f_{k}=\sum_{\left(\mathbf{e}_{k^{\prime}}-\mathbf{u}_{w}\right) \cdot \mathbf{n}<0}\left|\left(\mathbf{e}_{k^{\prime}}-\mathbf{u}_{w}\right) \cdot \mathbf{n}\right| \\
R_{f}\left(\mathbf{e}_{k^{\prime}} \rightarrow \mathbf{e}_{k}\right) f_{k^{\prime}}
\end{array}
$$

where $k^{\prime}$ and $k$ are the incident and reflected directions of the particles, $\mathbf{u}_{w}$ and $\rho_{w}$ are the velocity and density at the wall, $\mathbf{n}$ is the unit normal and $R_{f}$ is the the scattering kernel given by

$$
R_{f}\left(\mathbf{e}_{k^{\prime}} \rightarrow \mathbf{e}_{k}\right)=\left.\frac{A_{N}}{\rho_{w}}\left[\left(\mathbf{e}_{k}-\mathbf{u}_{w}\right) \cdot \mathbf{n}\right] f_{k}^{e q}\right|_{\mathbf{u}=\mathbf{u}_{w}} .
$$

The coefficient, $A_{N}$, can be evaluated from

$$
A_{N}=\rho_{w} \frac{\sum_{k}\left|\left(\mathbf{e}_{k}-\mathbf{u}_{w}\right) \cdot \mathbf{n}\right| f_{k}}{\left.\left|\left(\mathbf{e}_{k}-\mathbf{u}_{w}\right) \cdot \mathbf{n}\right| f_{k}^{e q}\right|_{\mathbf{u}=\mathbf{u}_{w}} \sum_{k}\left|\left(\mathbf{e}_{k^{\prime}}-\mathbf{u}_{w}\right) \cdot \mathbf{n}\right| f_{k^{\prime}}} .
$$

The Maxwellian diffuse reflection boundary condition assumes the reflected molecules are in thermal equilibrium with the wall, and therefore the energy density distribution function can be determined from

$$
g_{k}=\frac{D R}{2} T_{w} f_{k}
$$

where $T_{w}$ is the temperature of the wall.

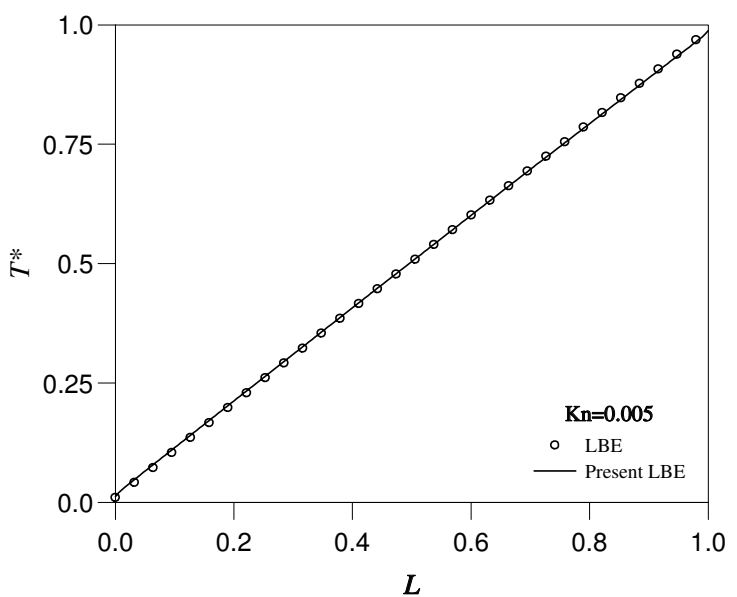

Fig. 2: Nondimensional temperature profiles for Fourier flow between two parallel plates at a Knudsen number of 0.005 . Comparison of the LBE solution with the Knudsen layer correction (-), and the LBE solution without the Knudsen layer correction (o).

The present model has been applied to rarefied Fourier flow between two horizontal parallel plates and the grid independent results are compared to the DSMC simulations reported by Gallis, Rader and Torczynski [31]. The 
temperatures of the upper and lower walls are assumed to be $T_{2}$ and $T_{1}$, respectively, and the reference temperature, $T_{\text {ref }}$, is $\left(T_{1}+T_{2}\right) / 2$. The nondimensional temperature is given by $T^{*}=\left(T-T_{1}\right) /\left(T_{2}-T_{1}\right)$, while the nondimensional distance is defined as $L=x / H$.

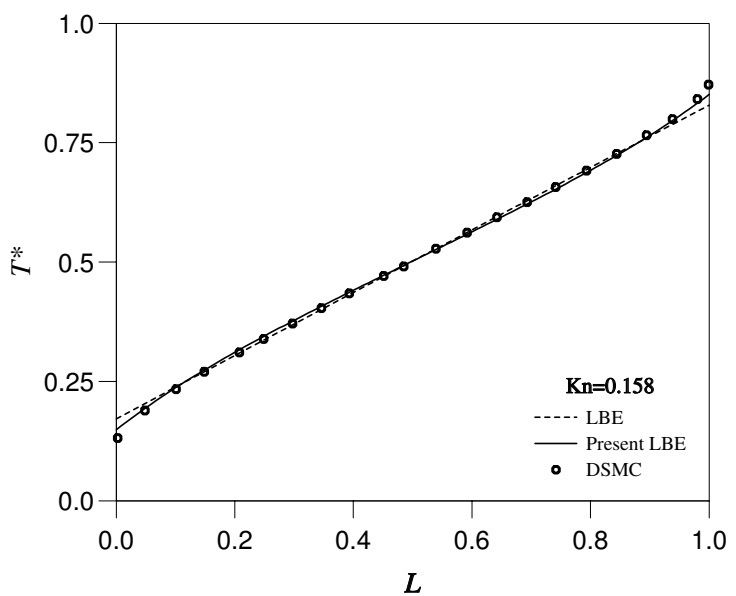

(a)

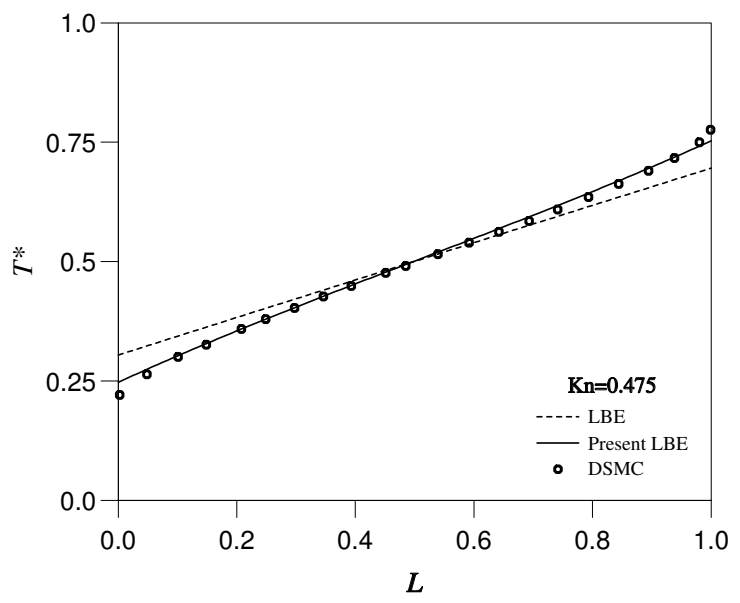

(b)

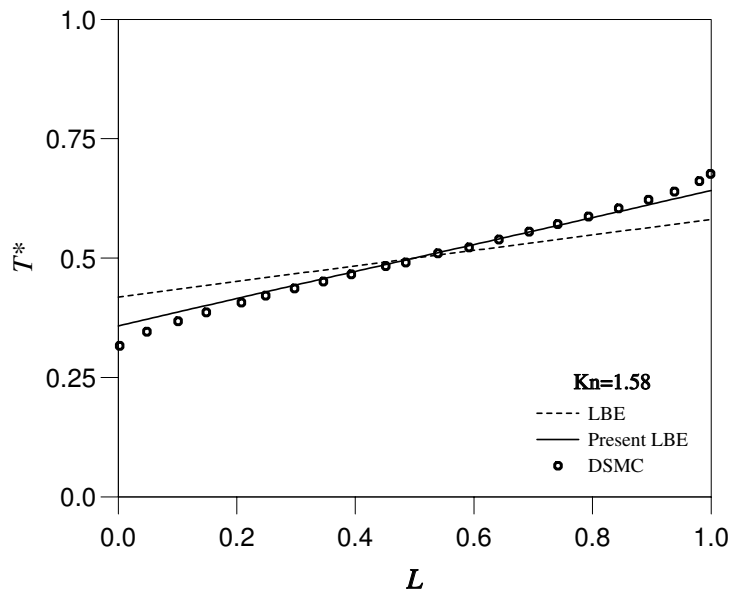

(c)

Fig. 3: Nondimensional temperature profiles for rarefied Fourier flow between two parallel plates using the Maxwellian gas molecular interaction model, i.e. $\omega=1.0$.
As $K n$ increases, the Knudsen layers attached to each plate will grow and will eventually start to overlap. Our LBE model assumes this effect to be additive. For example, if the distance between the parallel plates is $H$, then the effective mean free path in the overlapping Knudsen layers is assumed to be

$$
l_{e}=\frac{l}{1+\psi(x / l)+\psi[(H-x) / l]},
$$

where $x$ is the distance from the lower wall and $H-x$ is the distance from the upper wall.

The effect of the Knudsen layer correction should be minimal when the Knudsen number is small and this is illustrated in fig. 2 which shows the temperature profiles at a Knudsen number of 0.005. As expected, the LBE predictions with and without the Knudsen layer correction are almost identical. Figures 3(a)-3(c) compare the LBE and DSMC predictions at Knudsen numbers of 0.158 , 0.475 and 1.58 , and illustrate the growing influence of the Knudsen layer in the transition regime. The temperature profiles without the Knudsen layer correction follow an almost linear variation between the plates. However, when the correction is incorporated into the LBE model, the accuracy of the predictions is significantly improved and the model is able to capture the nonlinear temperature profile within the Knudsen layer. Even at a Knudsen number as high as 1.58 , the results are in good qualitative agreement with the DSMC data but the computational cost is significantly less.

In conclusion, the Knudsen layer correction for thermal LBE models offers an effective approach for the simulation of nonisothermal rarefied gas flow in micro- and nano-scale devices. The proposed technique has been successfully applied to Fourier flow and offers the computational efficiency of NSF methods but with a qualitative accuracy comparable to DSMC simulations. In this letter, we have used a new wall function concept which modifies the local mean free path to take into account the nonequilibrium effects in the Knudsen layer. In the next step, the model will be applied to more complex problems involving thermal and pressure-driven rarefied flows in the transition regime where the NSF equations fail to capture the observed nonequilibrium phenomena.

$$
* * *
$$

We would like to thank Dr. Michael Gallis of Sandia National Laboratories for kindly providing the DSMC data. This work was financially supported by the UK Engineering and Physical Sciences Research Council under grant no. GR/S77196/01 and additional support was provided by Collaborative Computational Project 12 (CCP12).

\section{REFERENCES}

[1] Benzi R., Succi S. and Vergassola M., Phys. Rep., 222 (1992) 145. 
[2] Chen S. and Doolen G. D., Ann. Rev. Fluid Mech., 30 (1998) 329.

[3] Succi S., The Lattice Boltzmann Equation for Fluid Dynamics and Beyond (Clarendon Press, Oxford) 2001.

[4] Wolf-Gladrow D. A., Lattice-gas Cellular Automata and Lattice Boltzmann Models: An Introduction (SpringerVerlag, Berlin) 2000.

[5] Lim C. Y., Shu C., Niu X. D. and Chew Y. T., Phys. Fluids, 14 (2002) 2299.

[6] Nie X. B., Doolen G. D. and Chen S., J. Stat. Phys., 107 (2002) 279.

[7] Niu X. D., Shu C. and Chew Y. T., Europhys. Lett., 67 (2004) 600.

[8] Lee T. and Lin C.-L., Phys. Rev. E, 71 (2005) 046706.

[9] Sbragaglia M. and Succi S., Phys. Fluids, 17 (2005) 093602.

[10] Sofonea V. and Sekerka R. F., J. Comp. Phys., 207 (2005) 639.

[11] Sofonea V. and Sekerka R. F., Phys. Rev. E, 71 (2005) 066709.

[12] Tang G. H., Tao W. Q. and He Y. L., Phys. Rev. E, 72 (2005) 056301.

[13] Toschi F. and Succi S., Europhys. Lett., 69 (2005) 549

[14] Zhang Y. H., Qin R. S. and Emerson D. R., Phys. Rev. E, 71 (2005) 047702.

[15] Benzi R., Biferale L., Sbragaglia M., Succi S. and Toschi F., J. Fluid Mech., 548 (2006) 257.

[16] Benzi R., Biferale L., Sbragaglia M., Succi S. and Toschi F., Europhys. Lett., 74 (2006) 651.

[17] Chikatamarla S. S., Ansumali S. and Karlin I. V., Europhys. Lett., 74 (2006) 215.

[18] Jeong N., Lin C.-L. and Choi D. H., J. Micromech. Microeng., 16 (2006) 1749.

[19] Sbragaglia M. and Succi S., Europhys. Lett., 73 (2006) 370.

[20] Zhang Y. H., Gu X. J., Barber R. W. and Emerson D. R., Phys. Rev. E, 74 (2006) 046704.

[21] Ansumali S., Karlin I. V., Frouzakis C. E. and Boulouchos K. B., Physica A, 359 (2006) 289.

[22] Zhang Y. H., Gu X. J., Barber R. W. and Emerson D. R., Daresbury Laboratory Technical Report, TR-2006002 (2006) .

[23] Shan X., Yuan X.-F. and Chen H., J. Fluid Mech., 550 (2006) 413.

[24] Guo Z. L., Zhao T. S. and Shi Y., J. Appl. Phys., 99 (2006) 074903.

[25] Chapman S. and Cowling T. G., The Mathematical Theory of Non-uniform Gases (Cambridge University Press, Cambridge) 1971.

[26] Lockerby D. A., Reese J. M. and Gallis M. A., Phys. Fluids, 17 (2005) 100609.

[27] Chen H., Kandasamy S., Orszag S., Shock R., Succi S. and Yakнot V., Science, 301 (2003) 633.

[28] He X., Chen S. and Doolen G. D., J. Comp. Phys., 146 (1998) 282.

[29] Shi Y., Zhao T. S. and Guo Z. L., Phys. Rev. E, 70 (2004) 066310.

[30] Zhang Y. H., Qin R. S., Sun Y. H., Barber R. W. and Emerson D. R., J. Stat. Phys., 121 (2005) 257.

[31] Gallis M. A., Rader D. J. and Torczynski J. R., Phys. Fluids, 14 (2002) 4290. 